\title{
Detection and characterisation of the genes encoding glyoxalase I and II from Neisseria meningitidis
}

\author{
GOKSEL KIZIL*, KATHY WILKS, D. WELLS and D. A. A. ALA'ALDEEN \\ Meningococcal Research Group, Divisions of Microbiology, School of Clinical Laboratory Sciences, University \\ of Nottingham Faculty of Medicine and Health Sciences, University Hospital, Nottingham NG7 2UH
}

\begin{abstract}
Glyoxalase enzymes I and II are involved in a detoxification process consisting of conversion of reactive dicarbonyl compounds (e.g., methylglyoxal) to less reactive hydroxy acids. The structural gene for meningococcal glyoxalase I (glo $A$ ) was identified by screening an expression library with a rabbit antiserum. The meningococcal glo $A$ gene consisted of 138 deduced amino acids, with a calculated mol. wt of $15.7 \mathrm{kDa}$. The DNA and deduced protein sequence of glo $A$ was compared to known sequences of glyoxalase I enzymes and showed high homology with glo $A$ of several eukaryotic and prokaryotic species. Insertion of a gloA-containing plasmid in Escherichia coli increased the host organism's tolerance to methylglyoxal from $<2 \mathrm{mM}$ to $>4 \mathrm{mM}$, thus demonstrating its functional identity. A databank search also revealed the presence of a putative gloB gene, encoding glyoxalase II (GIxII), in the recently released genomic sequences of Neisseria meningitidis and $N$. gonorrhoeae.
\end{abstract}

\section{Introduction}

Glyoxalase I (GlxI; $S$-D-lactoylglutathione methylglyoxal lysase) and glyoxalase II (GlxII; S-2-hydroxyacylglutathione hydrolase) are the enzyme components of the glyoxalase system. GlxI catalyses the formation of $S$-D-lactoylglutathione from hemithioacetal, formed non-enzymically or enzymically from methylglyoxal (2-oxo-aldehydes) and reduced glutathione, as shown in Fig. 1 [1]. GlxII catalyses the hydrolysis of $S$-Dlactoglutathione to D-lactic acid and regenerates reduced glutathione as shown in Fig. 1.

The physiological role of the glyoxalase system is still not completely defined, but it is evident that it represents a detoxification mechanism that inactivates the electrophilic 2-oxo-aldehydes. Many compounds, both aliphatic and aromatic, containing the di-carbonyl function have been tested and found to serve as substrates for the enzyme. The primary physiological

Received 4 Sept. 1999; revised version received 20 Nov. 1999; accepted 25 Nov. 1999.

Corresponding author: Dr D. A. A. Ala'Aldeen (e-mail: daa@nottingham.ac.uk).

* Present address: Chemistry Department, Faculty of Science and Art, University of Dicle, 21280 Diyarbakir, Turkey. substrate of the glyoxalase system is methylglyoxal (MG). The latter is formed non-enzymically from dihydroxyacetone phosphate and glyceraldehyde-3phosphate [2]

GlxI has been found in a wide variety of species, including mammals, prokaryotes and plants. The molecular mass of the enzyme from the different sources varies between 20 and $48 \mathrm{kDa}$. The enzyme derived from yeasts and bacteria appears to be monomeric, whereas the mammalian enzyme is a dimer with two identical subunits. The active site of GlxI contains a glutathione recognition site, general base catalyst and a proximate zinc ion, $\mathrm{Zn}^{2+}$ [3]. GlxII has also been found in most tissues of mammals, as well as in bacteria and plant species. To date, neither of these two enzymes has been reported in meningococci. This report describes the detection and characterisation of GlxI and a putative GlxII of Neisseria meningitidis following the screening of a genomic expression library.

\section{Materials and methods}

The methods for bacterial growth, genetic library screening, DNA sequencing and analysis were as described by Kizil et al. [4]. 

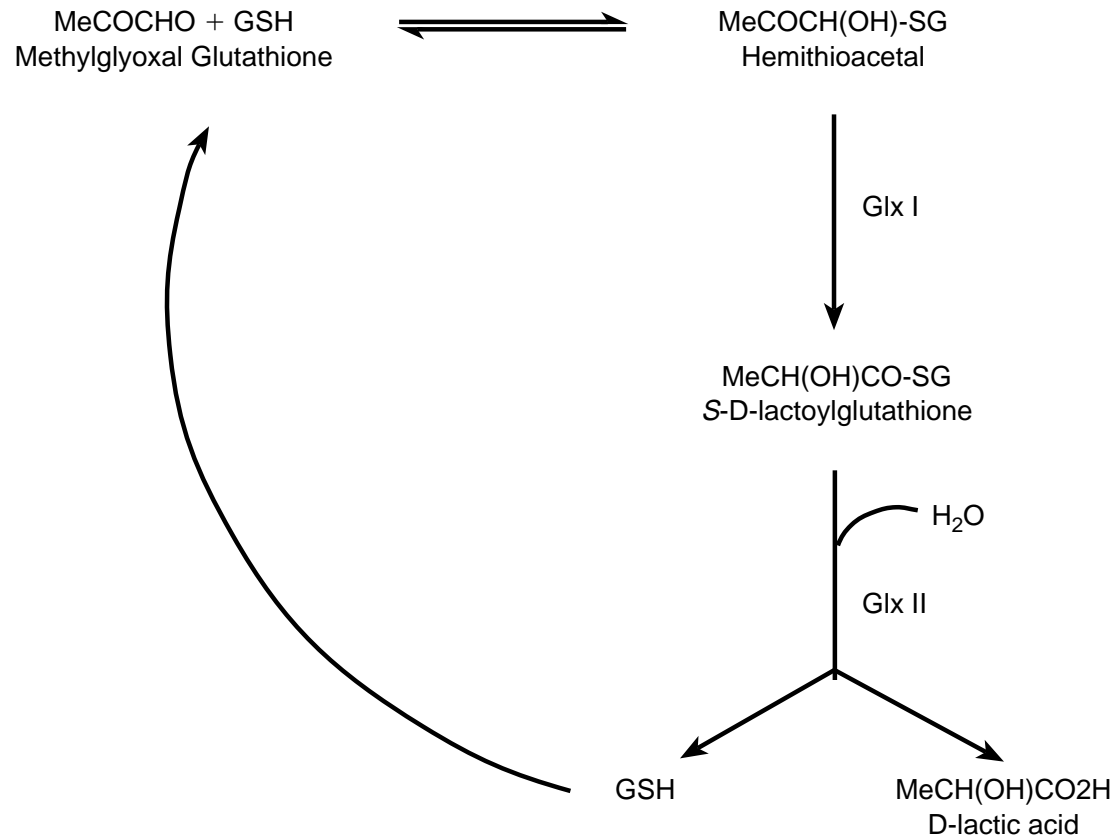

Fig. 1. Detoxification of methylglyoxal.

\section{Assay for glyoxalase I activity}

A stock solution of $1 \mathrm{M}$ methylglyoxal (MG; Sigma) was prepared in Luria Bertani Broth (LBB; Oxoid) and filtered through a $0.45-\mu \mathrm{M}$ filter. A series of $180-\mu \mathrm{l}$ volumes of LBB, containing increasing concentrations of $(0-16 \mathrm{mM})$ of methylglyoxal, was put in rows of a 96-well microtitration plate. A $20-\mu 1$ inoculum of each overnight culture $\left(10^{8}\right.$ organism $\left./ \mathrm{ml}\right)$ was added to each of these and incubated at $37^{\circ} \mathrm{C}$ (stationary). These Escherichia coli were transformants containing pBluescript with or without meningococcal gloA. At different time intervals, 20- $\mu 1$ samples were taken in duplicate from each well, diluted $\left(10^{1}-10^{4}\right)$ in phosphated-buffered saline (PBS) and $20-\mu l$ volumes of each dilution were seeded on to blood agar. The plates were incubated overnight, then the numbers of $\mathrm{cfu} / \mathrm{ml}$ were counted and the $\log _{10} \mathrm{cfu}$ of the average of the duplicates was documented. Where a clear reduction in cfu counts was detected, the test was repeated to obtain six cfu counts and their $\mathrm{p}$ value was calculated by a Sign test, as described previously [5].

\section{Results and discussion}

In a recent investigation, a meningococcal expression library was screened in this laboratory with rabbit polyclonal antibodies raised against meningococcal proteins [4]. Several clones of recombinant DNA were identified and rescued, in vivo, into pBluescript plasmids and fully sequenced. One of these meningococcal DNA fragments (clone $\mathrm{C} 1$ ) contained an open reading frame (ORF) that showed a high degree of DNA and deduced amino-acid homology with the gloA gene of several bacterial and eukaryotic species [4]. This ORF (named meningococcal gloA) consisted of
138 deduced amino acids with an estimated mol. wt of $15.7 \mathrm{kDa}$ (acc. no. Y14298). The deduced protein sequence showed homology with GlxI of Salmonella typhimurium (67\%) [6]. Lycopersicon esculentum (52\%) [7], Pseudomonas putida (50\%) [8] and Homo sapiens (47\%) [9]. Fig. 2 shows the protein sequence alignment of the GlxI of these organisms, which confirms the presence of several regions of sequence identity among them. These regions were analysed by a Basic Local Alignment Search Tool (BLAST) search of the NCBI non-redundant database, with the default parameters [10].

It was important to confirm the functional attributes of the identified meningococcal gene. Therefore, the effect of the meningococcal gloA gene on the sensitivity of an E. coli strain (XL1-Blue) to methylglyoxal (MG) was tested. As shown in Fig. 3a, the growth rate of $E$. coli containing pBluescript plasmid (no meningococcal DNA insert, negative control) was inhibited in the presence of $\mathrm{MG}$ in the medium. $\mathrm{MG}$ at $4 \mathrm{mM}$ and $16 \mathrm{mM}$ concentrations completely inhibited the growth of the cells after 2 and $1 \mathrm{~h}$, respectively, with no subsequent recovery. In the presence of $2 \mathrm{mM}$ MG, $\log _{10}>4$ growth inhibition was observed in the first $3 \mathrm{~h}$, which was sustained over $24 \mathrm{~h}$.

The E. coli strain transformed with pSKC1 (containing meningococcal $g l o A$ ), showed greater resistance to MG (Fig. 3b). In $2 \mathrm{~mm} \mathrm{MG}$, there was very little, if any, reduction in growth rate. At a concentration of $4 \mathrm{mM}$ MG, there was an early reduction of $\log _{10}>3$ in growth rate $(p<0.01)$, which recovered during the subsequent $22 \mathrm{~h}$, reaching levels comparable to the control $\left(\log _{10}<1\right.$, below control). At $16 \mathrm{mM}$ MG, growth reached complete inhibition very rapidly (within $2 \mathrm{~h})(\mathrm{p}<0.001)$. However, growth was observed to 


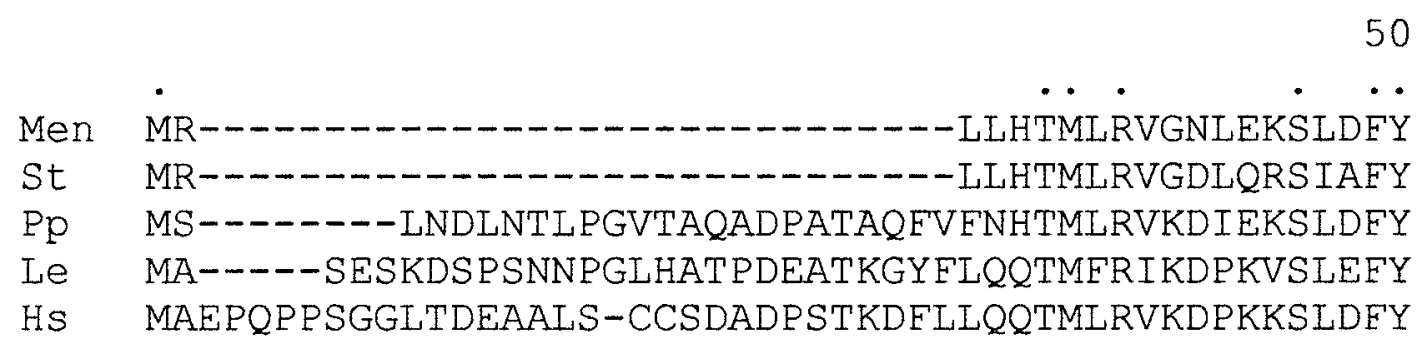

Men QNVLGMKLLRRKDYPEGRETLAEVGYGDETD-- - - - - - - STVL

St TNVLGMKLIRTSENPEYKYSLAFVGYGPETE------------EAVI

PP RRVLGEKLVDKRDFVEAKESLYELALVDPATIPADDDARHQWMKS I PGVL

Le SKVLGMSLLKRLDFPEMKFSLYFMGYEDTASAPSDPVERTAWTFSQKSTL

HS TRVLGMTLIQKCDEPIMKFSLYFLAYEDKNDIPKEKDEKIAWALSRKATL

Men ELTHNWDTER-----ŸDLGNA----ŸHIAVEVDDAYEACERVKRQGGN

St ELTYNWGVES-----YDMGNA---YGHIGLSVDNAAEACERIRQNGGN

Pp ELTHNHGTERDADFA-YHHGNTDPRGFGHICVSVPDVVAACEREEALQVP

Le ELTHNWGTESDPNFTGYHNGNSEPRGFGHIGVTVDDVYKACERFESLGVE

HS ELTHNWGTEDDATQS-YHNGNSDPRGFGHIGIAVPDVYSACKREEELGVK

\section{3}

$\begin{array}{ll}\text { Men } & \text { VVREAGPMKHGTTVIAFVEDPDGYKIEFIQKKSGDDSVAYQTA } \\ \text { St } & \text { VTREAGPVKGGSTIIAFVEDPDGYKIELIEAKDAGRGLG---N } \\ \text { Pp } & \text { FQKR--LSDGRMNHLAFIKDPDGYWVEIQP-------TPL } \\ \text { Le } & \text { FVKK--PLDGKMKGIAFIKDPDGYWIEIFDTKIIKDAAG-SAS } \\ \text { HS } & \text { EVKK--PDDGKMKGLAFIODPDGYWIEILNPNKM----A-TLM }\end{array}$

Fig. 2. GlxI amino-acid sequence alignment of N. meningitidis (Men, acc. no. Y14298), S. typhimurium (St, U57364), P. putida (Pp, D00342), L. esculentum (Le, Z48183) and H. sapiens (Hs, AF146651). The relatively conserved catalytic loop is underlined, catalytic base is italicised, and those residues printed in bold type represent the putative $\mathrm{Zn}^{2+}$ binding ligands.

recover transiently during the next hour, but this recovery was not sustained (Fig. 3b).

In additon to glo $A, \mathrm{pSKC} 1$ also contained a truncated tspA gene (downstream of gloA) [4]. To rule out the possibility of TspA being responsible for the resistance to $\mathrm{MG}$, the truncated $t \operatorname{sp} A$ was removed by digesting pSKC1 with Sal I enzyme (Strategene) which happened to cut on both sides of the gene. The open plasmid (pSKC1-TR) was then re-ligated using T4 DNA ligase (Boehringer-Mannheim). E. coli transformed with pSKC1-TR showed the same level of tolerance to MG as pSKC1 (data not shown).
Based on sequence comparison, GlxI enzyme appeared to contain a flexible peptide loop near the active site that is structurally (and functionally) analogous to the catalytic loop of chicken muscle triosephosphate isomerase (TIM) [11]. This region includes residues 100-111 in man, which correspond to residues 56-65 in meningococci. Furthermore, Glu-100 of human GlxI (corresponding to Glu-56 in meningococci) might be the active site base involved in the enediol-protontransfer mechanism proposed for GlxI enzyme.

GlxI is dependent on bivalent metal ions for catalytic activity. The essential metal of the native enzyme has 


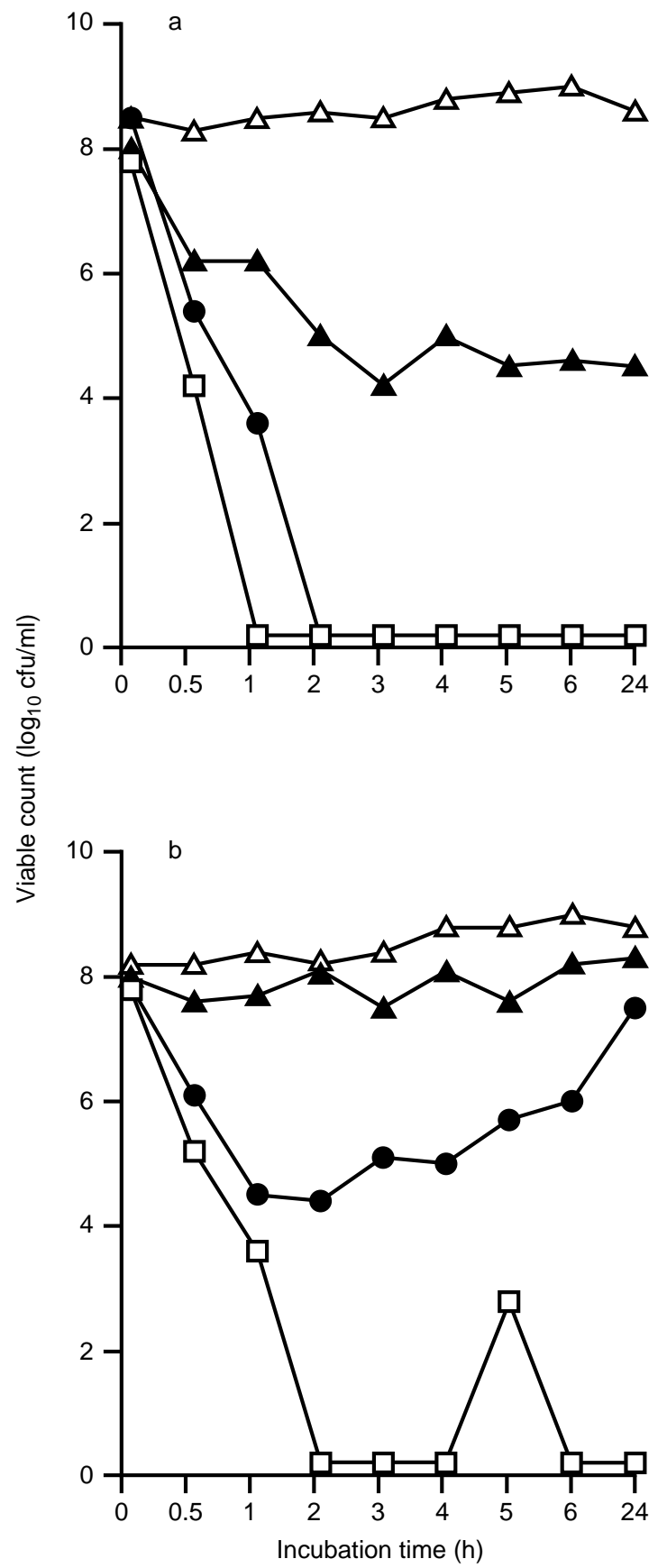

Fig. 3. Growth curve of E. coli strain XLI-Blue containing (a) pBluescript (negative control) or (b) pSKC1 in the presence of increasing concentrations of methylglyoxal; $\triangle$, no MG (negative control); $\boldsymbol{\Delta}, 2 \mathrm{mM} ; \bullet$, $4 \mathrm{mM}$; $\square, 16 \mathrm{mM}$.

been identified as zinc [12]. Previous studies also suggest that $\mathrm{Zn}^{2+}$ appears to bind three-to-five (often four) co-ordination ligands, and the types of ligands consist of histidine, cysteine, glutamate, aspartate or water [13]. Clugston et al. [6] also identified possible zinc-binding residues, based on the available GlxI sequences and their alignments. In $S$. typhimurium $\mathrm{Zn}^{2+}$ ligands were identified as His-74, Cys-86, Asp115, Asp-117 and Glu-122 [6], which appear to be conserved in meningococci (Fig. 2).

The amino-acid sequence of the meningococcal GlxI was also used to search the genomic sequence of $N$. gonorrhoeae (University of Oklahoma, Ok, USA) and a putative GlxI with $97 \%$ (395 of 406) identity to meningococcal GlxI, was identified.

It was of interest to see whether $N$. meningitidis possesses $g l o B$, the gene encoding GlxII. The first gloB gene was isolated from human liver and had a mol. wt of $28 \mathrm{kDa}$ [14]. Recently, a gloB gene was also identified and characterised in the yeast Saccharomyces cerevisiae, with a mol. wt of $31 \mathrm{kDa}$ and $59 \%$ identity at protein level to its human counterpart [15]. Here, the human GlxII amino-acid sequence was obtained and used to search the genomic sequence data released for N. meningitidis (Sanger, Wellcome). A putative gloB gene was identified with 250 deduced amino acids and an estimated mol. wt of $27.8 \mathrm{kDa}$. Again, a high level of conservation was observed and the deduced protein showed 35\% identity with human GlxII. The functional attributes of this gene can be determined following cloning and expression of biologically active forms of the encoding protein and tested in an isolated system, such as E. coli. However, ideally, the function of GlxI and GlxII would be better characterised by testing the ability of isogenic gloA and gloB deletion mutants of $N$. meningitidis to survive increasing concentrations of methylglyoxal, compared to the wild-type strain. These experiments were beyond the objectives of the present study. In conclusion, this is the first identification of the presence of the glyoxalase system in $N$. meningitidis and N. gonorrhoeae.

We thank the University of Dicle, Diyarbakir, Turkey, for providing a studentship grant to G. K.

\section{References}

1. Thornalley PJ. The glyoxalase system: new developments towards functional characterization of a metabolic pathway fundamental to biological life. Biochem $J$ 1990; 269: 1-11.

2. Thornalley PJ. Pharmacology of methylglyoxal: formation, modification of proteins and nucleic acids, and enzymatic detoxification: a role in pathogenesis and antiproliferative chemotherapy. Gen Pharmacol 1996; 27: 565-573.

3. Allen RE, Lo TWC, Thornalley PJ. Inhibitors of glyoxalase I: design, synthesis, inhibitory characteristics and biological evaluation. Biochem Soc Trans 1993; 21: 535-540.

4. Kizil G, Todd I, Atta M, Borriello SP, Ait-Tahar, Ala'Aldeen DAA. Identification and characterization of TspA, a major CD4+ T-cell- B-cell-stimulating Neisseria-specific antigen. Infect Immun 1999; 67: 3533-3541.

5. Ala'Aldeen DAA, Borriello SP. The meningococcal transferrinbinding proteins 1 and 2 are both surface exposed and generate bactericidal antibodies capable of killing homologous and heterologous strains. Vaccine 1996; 14: 49-53.

6. Clugston SL, Daub E, Kinach R, Miedema D, Barnard JF, Honek JF. Isolation and sequencing of a gene encoding for glyoxalase I activity from Salmonella typhimurium and comparision with other glyoxalase I sequences. Gene 1997; 186: $103-111$.

7. Espartero J, Sanches-Aguayo I, Pardo JM. Molecular characterization of glyoxalase-1 from a higher plant; upregulation by stress. Plant Mol Biol 1995; 29: 1223-1233.

8. Rhee H, Murata K, Kimura A. Purification and characterization of glyoxalase I from Pseudomonas putida. Biochem Biophys Res Commun 1986; 141: 993-999.

9. Kim N-S, Umezawa Y, Ohmura S, Kato S. Human glyoxalase I. cDNA cloning, expression, and sequence similarity to 
glyoxalase I from Pseudomonas putida. J Biol Chem 1993; 268: $11217-11221$.

10. Altschul SF, Gish W, Miller W, Myers WE, Lipman DJ. Basic Local Alignment Search Tool. J Mol Biol 1990 215: 403-410.

11. Lan $\mathrm{Y}, \mathrm{Lu} \mathrm{T}$, Lovett PS, Creighton DJ. Evidence for a (triosephosphate isomerase-like) "catalytic loop" near the active site of glyoxalase I. $J$ Biol Chem 1995; 270: $12957-12960$.

12. Sellin S, Rosevear PR, Mannervik B, Mildvan AS Nuclear relaxation studies of the role of the essential metal in glyoxalase I. $J$ Biol Chem 1982; 257: $10023-10029$.
13. Jernigan R, Raghunathan G, Bahar I. Characterization of interactions and metal ion binding sites in proteins. Curr Opin Struct Biol 1994; 4: 256-263.

14. Ridderström M, Saccucci F, Hellman U, Bergman T. Principato G, Mannervik B. Molecular cloning, heterologous expression, and characterization of human glyoxalase II. $J$ Biol Chem 1996; 271: 319-323.

15. Bito A, Haider M, Hadler I, Breitenbach M. Identification and phenotypic analysis of two glyoxalase II encoding genes from Saccharomyces cerevisiae, GLO2 and GLO4, and intracellular localizaton of the corresponding proteins. J Biol Chem 1997; 272: $21509-21519$ 